\title{
Inhibition by phospholipids of haemolytic action of asbestos
}

\author{
M. C. JAURAND, L. MAGNE, AND J. BIGNON \\ From the Laboratoire de Biopathologie Pulmonaire, INSERM 139, Hôpital Henri Mondor, 51, \\ Avenue du Maréchal de Lattre de Tassigny, 94000 Créteil, France
}

ABSTRACT Haemolysis by asbestos fibres results from an increase in membrane permeability and not from rupture of red blood cells (RBC). The effect of chrysotile asbestos on RBC is at least partly, if not completely, attributable to lipid extraction and adsorption on to the fibres. This was suggested by the hyperbolic relationship between the haemolytic activity of chrysotile and the relative concentration of both chrysotile and RBC. Moreover, it was shown that pre-incubation of chrysotile with lipids, either as RBC membranes or with pure lipids in the form of liposomes, prevents haemolysis.

Mineral fibres of the asbestos group are known to have a fibrogenic and carcinogenic or cocarcinogenic effect in humans. The mechanism of their carcinogenicity is still not established and it is not known whether it is related to the chemical or to the physical properties of the fibres. The type of asbestos most used by industry is chrysotile. Previous studies have suggested that chrysotile fibres interact with cell membranes; Macnab and Harington (1967), and Beck et al. (1972) demonstrated in vitro that they induce haemolysis of red blood cells (RBC) and release lactate dehydrogenase from macrophages. Macnab and Harington (1967) indicated that membrane glycoproteins may interact with chrysotile; in this paper, it is shown that lipids do likewise, because pre-treatment of chrysotile fibres with either RBC membranes or with liposomes results in a lower rate of haemolysis. The interaction of chrysotile with lipids can greatly modify the integrity and stability of cell membranes or of the pulmonary surfactant, and changes their physical properties.

\section{Materials and methods}

All experiments were carried out with UICC samples of chrysotile and crocidolite. Granulometric electron microscopy studies showed that the chrysotile fibres were on average less than $5 \mu \mathrm{m}$ in length; the crocidolite was less than $4 \mu \mathrm{m}$. Suspensions of the

Received for publication 17 April 1978

Accepted for publication 6 July 1978 fibres were obtained by ultrasonic treatment at $20 \mathrm{kHz}$ for $45 \mathrm{~min}$ in isotonic veronal buffer of $\mathrm{pH}$ $7 \cdot 26$.

Human blood was collected in citrate; RBC were washed three times with isotonic saline and resuspended in veronal buffer. Fibres and RBC suspensions were pre-incubated for $10 \mathrm{~min}$ at $37^{\circ} \mathrm{C}$ (finalconcentration $\times 2$ ). Then $1 \mathrm{ml}$ aliquots of each suspension were mixed together and incubated for various periods at $37^{\circ} \mathrm{C}$. At the end of the reaction the samples were centrifuged $(10 \mathrm{~min}, 1200 \mathrm{~g})$. The optical density of the supernatant was determined at $540 \mathrm{~nm}$. Results are expressed as percent of haemolysis. Complete haemolysis was obtained by the addition of $0.2 \% \mathrm{v} / \mathrm{v}$ Triton $\mathrm{X}-100$ to the $\mathrm{RBC}$ suspension.

In order to have the same unit to express fibres and RBC concentrations, conversions in terms of surface were used because it is assumed that the adsorption phenomenon of molecules, such as phospholipids engaged in an organised structure (RBC membranes), must be related to the extent of the surface yielded by both molecules and adsorbent. The specific surface area of chrysotile was taken to be $25 \mathrm{~m}^{2} \times \mathrm{g}^{-1}$ measured by $\mathrm{N}_{2}$ adsorption (Data sheets of physical and chemical properties of UICC standard reference asbestos samples (1971); and unpublished data from our laboratory). The RBC surface $\left(92 \mu \mathrm{m}^{2}\right)$ was estimated from the radius of a sphere assumed to have a volume of $87 \mu \mathrm{m}^{3}$ (Altman and Dittmer, 1974).

The inhibition of haemolysis was obtained by 
pre-incubation of chrysotile with RBC membranes, in the proportion of $0.022-0.25 \mathrm{mg}$ lipids per $\mathrm{mg}$ chrysotile, or with liposomes. RBC membranes were obtained by lysis in distilled water. These membranes, suspended in veronal buffer, were stirred into the chrysotile. The total lipid content of human $\mathrm{RBC}$ was taken as $5 \cdot 1 \mathrm{mg} \mathrm{ml}^{-1}$ (Altman and Dittmer, 1974). Liposomes were prepared as described by Papahadjopoulos and Watkins (1967), with dipalmitoyl phosphatidyl choline (DPL) (Calbiochem) alone, or with DPL, cholesterol and dicetylphosphate (DCP) in a molar ratio of $7: 2: 1$. For this experiment, asbestos fibres were pre-incubated as described above.

\section{Results}

Previous studies on the kinetics of RBC lysis by asbestos fibres followed on the one hand by $\mathrm{K}^{+}$and haemoglobin release (Jaurand et al., 1978), and on the other hand by phase contrast microscopy, have shown that the effect of asbestos on RBC is not a rupture of the cell but a progressive increase in the membrane permeability. Phase contrast microscopy shows that, when in contact with chrysotile fibres, RBC progressively increase their permeability; this was seen as a progressive decrease in contrast. The cells lose haemoglobin, gradually become ghosts, and subsequently the ghosts themselves disappear into the bundles of fibres. Moreover, quantitative

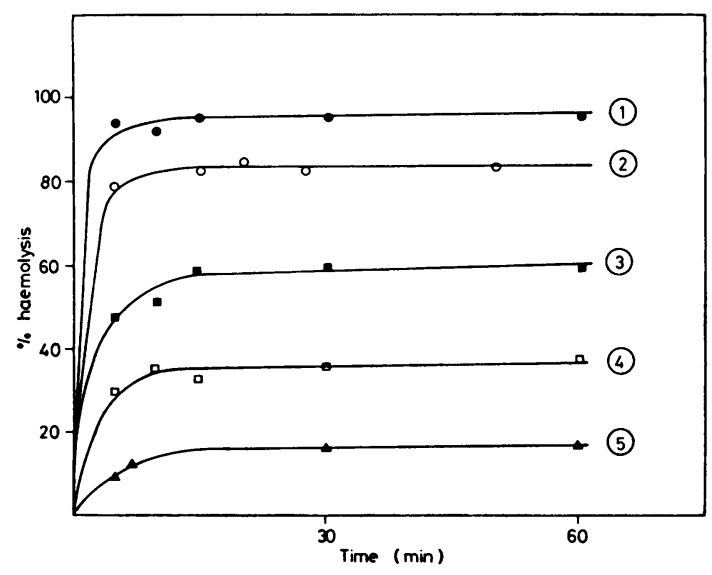

Fig. 1 Kinetics of haemolysis of human RBC by UICC A chrysotile (Ch) and crocidolite. Final concentrations: (l) Ch $5 \mathrm{mg} \mathrm{ml}^{-1}, \mathrm{RBC} 5 \%$ or Ch $0.5 \mathrm{mg} \mathrm{ml}^{-1}$; RBC 0.5\%; (2) Ch $0.22 \mathrm{mg} \mathrm{ml}^{-1}$, RBC $0.5 \%$; (3) Ch $1 \mathrm{mg} \mathrm{ml} \mathrm{m}^{-1}, \mathrm{RBC} 5 \%$ or $\mathrm{Ch}$ ' $0.1 \mathrm{mg} \mathrm{ml}^{-1}, \mathrm{RBC} 0.5 \%$; (4) Ch $0.5 \mathrm{mg} \mathrm{ml}^{-1}$, $R B C 5 \%$; (5) crocidolite $1 \mathrm{mg} \mathrm{ml}^{-1}, R B C 1 \%$. analyses have shown that the $\mathrm{K}^{+}$ion is released first, followed by haemoglobin.

Figure 1 shows the kinetics of haemolysis in an isotonic medium with various concentrations of RBC and fibres. The level of haemolysis, expressed as percent of total haemolysis obtained by addition of Triton X-100, is not always complete. The maximal haemolysis reached after 5-15 min contact depends on the relative concentration of chrysotile and $\mathrm{RBC}$, but not on the absolute concentration. For a given proportion of chrysotile and cells, a ten-fold increase in the absolute concentration re. sults in the same kinetics and final level of haemoly. sis (Figure 1, curves 1 and 3).

It appears that there is a clear relationship between the maximal haemolysis and the relative surface of chrysotile and RBC in the medium. When the amounts of chrysotile and $\mathrm{RBC}$ are expressed in terms of their relative active surfaces, and maximal haemolysis is plotted against the ratio (R) chrysotile surface:RBC surface, an excellent hyperbolic relationship is obtained (Figure 2). The inverse representation shows that $50 \%$ haemolysis is obtained at $R=0 \cdot 32$. Complete haemolysis occurs at $\mathrm{R}=2 \cdot 5$. Various concentrations of both $\mathrm{RBC}$ and chrysotile were tested keeping this ratio constant and $100 \%$ haemolysis was always found. This means that complete haemolysis is obtained only with a large excess of chrysotile surface. This result is consistent with the observation of Schnitzer and Pundsack (1970) on the dependence of haemolytic activity upon the surface area or degree of opening

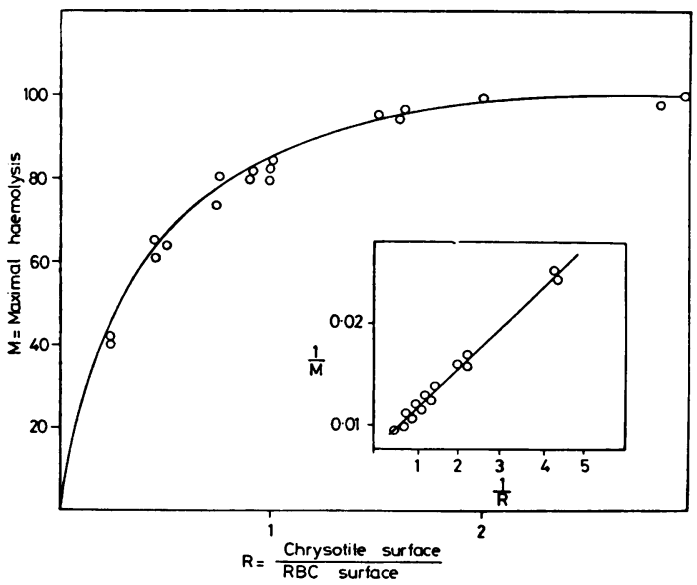

Fig. 2 Variation of maximal haemolysis $(M)$ with ratio $(R)$ of chrysotile/RBC surfaces. In insert is plotted the relationship:

$$
\frac{1}{M}=f\left(\frac{I}{R}\right)
$$


of the fibres. Therefore it may be assumed that the inhibition of the haemolytic effect of the fibre can be attributable to binding to RBC membrane components, either lipids or proteins, or both. In order to check this hypothesis, haemolytic experiments were carried out with chrysotile fibres pre-incubated with either RBC ghosts or with pure lipids.

Given amounts of chrysotile fibres were mixed with various amounts of RBC ghosts or liposomes made either of dipalmitoyl phosphatidyl choline (DPL) or of a mixture of DPL, dicetyl phosphate and cholesterol. After some minutes of contact, RBC were added to the mixture. The maximal lysis was measured and compared with the maximal lysis in experiments with untreated fibres. Figure 3 shows the inhibition of haemolysis expressed as the difference between maximal haemolysis by treated and untreated chrysotile (percentage of the value obtained with untreated) against the amount of lipids, either as ghosts or liposomes, added per mg of chrysotile. The pre-incubation of fibres, either with ghosts or liposomes, prevents subsequent haemolysis of RBC. This protective effect of preincubation is not caused by a mechanical effect of the presence of added particles, because the addition of latex particles to a chrysotile suspension to mimic

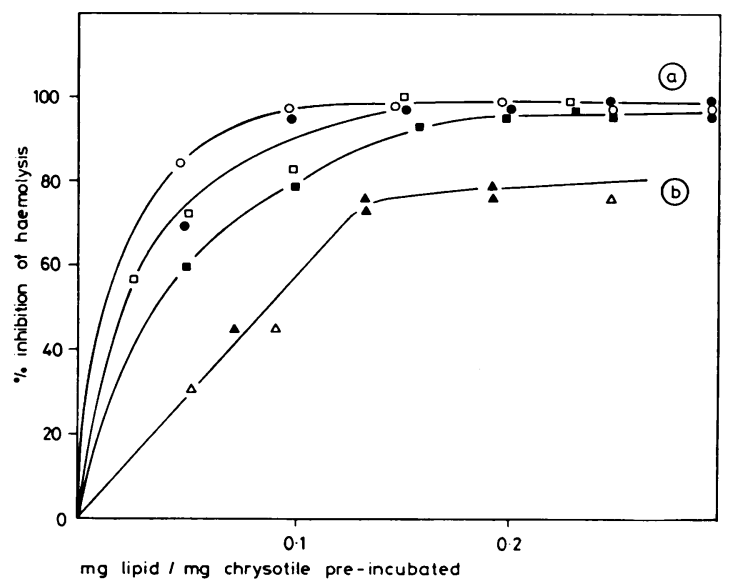

Fig. 3 Inhibition of haemolysis by pre-incubation of chrysotile $(C h)$ with $R B C$ membranes. Curves a:

(O) $R=0.2$, Ch $44 \mu g \mathrm{ml}^{-1}, \mathrm{RBC} 0.5 \%$;

(O) $R=0.3$, Ch $65 \mu \mathrm{g} \mathrm{ml} \mathrm{l}^{-1}, \mathrm{RBC} 0.5 \%$;

(口) $R=0.45$, Ch $1 \mathrm{mg} \mathrm{ml} \mathrm{m}^{-1}, \mathrm{RBC} 5 \%$;

(घ) $R=0.60$, Ch $130 \mu \mathrm{g} \mathrm{ml}^{-1}, \mathrm{RBC} 0.5 \%$.

Curve $b$ : Inhibition of haemolysis by pre-incubation

of chrysotile (Ch) with liposomes. Kinetics were

obtained with $1 \mathrm{mg} \mathrm{ml}^{-1} \mathrm{Ch}$ and $5 \% \mathrm{RBC}$.

(A) dipalmitoyl phosphatidyl choline liposomes,

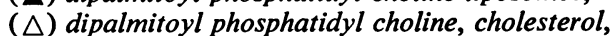
dicetyl phosphate liposomes. the presence of ghosts or liposomes in the reaction medium did not modify haemolysis. Moreover, when RBC were pre-incubated with ghosts or liposomes and then added to untreated chrysotile, the inhibition effect was decreased. When chrysotile was pre-incubated with the amount of RBC membrane corresponding to that of red cells lysed at the maximal haemolysis reached with untreated fibres, $95-100 \%$ inhibition was obtained.

\section{Discussion}

Kinetic studies have shown the dependence of haemolysis on the relative concentrations of both chrysotile and $\mathrm{RBC}$. The results indicate that $\mathrm{RBC}$ haemolysis by chrysotile is a self-inhibiting process.

Microscopic and kinetic observations showed that the effect of asbestos on RBC is to increase the membrane permeability. Such an effect is at least partly, if not completely, caused by lipid extraction and adsorption on to the fibres.

The protective effect appears mainly to be attributable to lipids, because the inhibition levels obtained with pure lipid are of comparable magnitude to the levels obtained with ghosts. This result corroborates the experimental work of Stalder and Stöber (1965), which led to the assumption that the lysis of red blood cells by silica and inert dust is caused by the adsorption of lipids from the red blood cells on to the surface of the dust. The result may also be compared to the concept proposed by Nash et al. (1966), that the highest interaction occurs between the hydroxyl group $(\mathrm{OH})$ of the silica surface and the negative charges of phospholipids. Thus, the $\mathrm{OH}$ group of the brucite layer of chrysotile may be important in the binding of membranes to chrysotile. If either $\mathrm{OH}$ or $\mathrm{Mg}^{2+}$ are (as assumed by Macnab and Harington, 1967) the major factors responsible for haemolysis by chrysotile, it follows that the haemolytic activity of chrysotile is principally determined by the surface charge of the fibres, the surface area being a secondary factor. The importance of surface charge was demonstrated by Light and Wei (1977) who found a correlation between $\zeta$ potential and haemolytic activity of asbestos; moreover, they found that both $\zeta$ potential and haemolytic activity were reduced by adding dipalmitoyl phosphatidyl choline. In their experiment, the phospholipid was not added in the form of liposomes used in the experiments described here as a model for membranes. However, there is a significant difference between ghosts and liposomes: the effect is greater with ghosts than with liposomes. For example, when haemolysis is performed with $5 \% \mathrm{RBC}$ and $1 \mathrm{mg} / \mathrm{ml}$ chrysotile pre-incubated with $0.18 \mathrm{mg}$ of lipids, only $80 \%$ inhibition is obtained 
when the lipids are added as pure phospholipid liposomes, compared with $99-100 \%$ inhibition obtained when the lipids are added as ghost lipids. This difference may be ascribed either to the fact that membrane components other than lipid are effective in inhibition, or to the chemical nature of the lipids used to prepare the liposomes, which differ from the membrane lipids.

Harington et al. (1971) and Desai et al. (1975) described the binding of haemoglobin to chrysotile. With the fibres used in our experiments this was not observed even when purified haemoglobin was used. It is noteworthy that chrysotile fibres are not the only ones capable of interacting with lipids. Haemolysis by crocidolite fibres is also inhibited by pre-incubation of the fibres with liposomes: no haemolysis was observed when $2 \mathrm{mg}$ of crocidolite fibres were pre-incubated with $0.41 \mathrm{mg}$ of dipalmitoyl phosphatidyl choline.

We thank Professor C. M. Gary Bobo for helpful discussions.

\section{References}

Altman, P. L., and Dittmer, D. S. (1974). Biology Data Book, vol. 3. Federation of American Societies of Experimenta Biology: Bethesda, Maryland.
Beck, E. G., Holt, P. F., and Manojlović, N. (1972). Comparison of effects on macrophage cultures of glass fibre, glass powder, and chrysotile asbestos. British Journal of Industrial Medicine, 29, 280-286.

Data sheets of physical and chemical properties of UICC standard reference asbestos samples (1971). Industrial Hygiene Department, National Research Institute for Occupational Diseases: Johannesburg, South Africa.

Desai, R., Hext, P., and Richards, R. (1975). The prevention of asbestos-induced hemolysis. Life Sciences, 16, 19311938.

Harington, J. S., Miller, K., and Macnab, G. (1971). Hemolysis by asbestos. Environmental Research, 4, 95-117.

Jaurand, M. C., Bignon, J., Gaudichet, A., Magne, L., and Oblin, A. (1978). Biological effects of chrysotile after $\mathrm{SO}_{2}$ sorption. II. Effects on alveolar macrophages and red blood cells. Environmental Research, 17, 216-227.

Light, W. G., and Wei, E. T. (1977). Surface charge and hemolytic activity of asbestos. Environmental Research, 13, 135-145.

Macnab, G., and Harington, J. S. (1967). Haemolytic activity of asbestos and other mineral dusts. Nature, 214 522-523.

Nash, T., Allison, A. C., and Harington, J. S. (1966). Physicochemical properties of silica in relation to its toxicity. Nature, 210, 259-261.

Papahadjopoulos, D., and Watkins, J. C. (1967). Phospholipid model membrane. II. Permeability properties of hydrated liquid crystals. Biochimica et biophysica acta, 135, 639-652.

Schnitzer, R. J., and Pundsack, F. L. (1970). Asbestos hemolysis. Environmental Research, 3, 1-13.

Stalder, K., and Stöber, W. (1965). Haemolytic activity of suspensions of different silica modifications and inert dusts. Nature, 207, 874-875. 